\title{
Perfeccionamiento de las instituciones políticas como posibilidad del desarrollo de la persona moral*
}

\section{Improvement of political institutions as a possibility for the development of moral persons}

\author{
Claudia Milena Rodríguez Valencia**
}

\begin{abstract}
*Artículo de investigación derivado de la tesis "Constitucionalización de los mínimos sociales desde la Teoría de la Justicia en John Rawls" de la Maestría en Filosofía de la Universidad Tecnológica de Pereira.

**Maestría en Filosofía, Especialista en Derecho Constitucional (Universidad del Rosario), abogada y filósofa. Directora Jurídica y Secretaria General de las Empresas Públicas de Armenia. milesofi0111@hotmail.com
\end{abstract}

Cómo citar: Rodríguez, C.M. (2018) Perfeccionamiento de las instituciones políticas como posibilidad del desarrollo de la persona moral. Inciso, 20(2); 14-26.

Recibido: 06/04/2018 Revisado: 17/07/2018Ａceptado: 10/12/2018

DOI: http://dx.doi.org/ 10.18634/incj.20v.2i.893

Resumen

La concepción igualitarista de Rawls tiene entre sus objetivos regular las desigualdades económicas y sociales, limitando las brechas de clase, la pobreza y las luchas por el cubrimiento de las necesidades básicas. Con el apoyo argumental de la justicia como equidad rawlsiana, se buscará ofrecer razones de peso sobre: Tendría que ser una de las funciones fundamentales del Estado garantizar el cubrimiento de necesidades básicas que le posibiliten una vida digna a todos sus coasociados o, en otras palabras, unas de las funciones del Estado tendrá que ser garantizar los derechos sociales fundamentales de todos sus coasociados.

La tesis se apoyará fundamentalmente en la teoría contractualista, ya que, se pretende hacer evidente el papel fundamental de las instituciones en el desarrollo de la persona moral. En esta instancia se asume que solo a través del Estado, se les pueden garantizar a los ciudadanos unos mínimos de bienestar que permitan cubrir sus necesidades básicas y en ese sentido, su participación política real y efectiva. Nos atrevemos a afirmar que en caso de su incumplimiento: "el Estado habrá roto el pacto", lo que a su vez tendría que implicar toda una serie de efectos a favor de los coasociados. Justamente, la doctrina del contrato social, en nuestros Estados modernos, se habrá de considerar como una de las más fuertes líneas argumentales en la filosofía política contemporánea y que coadyuva a conferirle legitimidad a todo sistema social y jurídico, así como deslegitimar estructuras sociales que no concuerdan con una fundamentación razonable y justa.

Palabras clave: Derechos sociales fundamentales, perfeccionamiento de las instituciones, desarrollo de la persona moral, dignidad humana, principios de justicia.
Rawls's egalitarian conception has among its objectives to regulate economic and social inequalities, limiting class gaps, poverty and the struggles for covering basic needs. With the argumentative support of justice as Rawlsian equity, we will try to offer strong reasons on (about the following statement): It would have to be one of the fundamental functions of the State to guarantee the covering of basic needs that allow a dignified life to all its co-associates or, in other words, one of the functions of the State would have to be to guarantee the fundamental social rights of all its co-associates.

The thesis will be fundamentally based on contractarian theory, since it is intended to make evident the fundamental role of institutions in the development of moral persons. In this instance, it is assumed that only through the State is how citizens can be guaranteed a minimum of well-being that covers their basic needs and, in that sense, their real and effective political participation. We dare to affirm that in case of non-compliance: "the State will have broken the pact", which in turn would have to involve a whole series of effects in favor of the co-associates. Precisely, the doctrine of the social contract, in our modern states, would have to be considered as one of the strongest argumentative lines in contemporary political philosophy, and which contributes to confer legitimacy on all social and legal systems, as well as to delegitimize social structures that do not agree with a reasonable and fair foundation.

Keywords: Fundamental social rights, improvement of institutions, development of moral persons, human dignity, principles of justice. 


\section{Introducción}

El problema de la filosofía política moderna se centró en la gran brecha que suscitaba el debate de liberales y comunitaristas, en cuanto los límites que impone el Estado frente al individuo, los valores comunes que se comparten en sociedad, la limitación de la libertad en procura de los mismos y la distribución equitativa del ingreso y la riqueza. Para Rawls, "Los debates a lo largo de los dos últimos siglos dejan claro que no hay un acuerdo básico sobre cómo han de organizarse las instituciones para favorecer mejor la libertad y la igualdad de la ciudadanía democrática" (Rawls, 2017: 24).

Sin embargo, en el siglo $\mathrm{XX}$, el problema de la filosofía moral y política se renueva con el que se ha considerado el más grande pensador político de Estados Unidos, a saber: John Rawls. Hasta entonces, muchos filósofos distinguían, por un lado, las pretensiones de libertad, y, por el otro, de lo justo, pero, además, cada uno de ellos le otorgaba cierta prioridad y valor absoluto a cada una de ellas en su contexto. No obstante, desde el punto de vista de Rawls, estas se pueden reconciliar bajo una justicia como equidad.

Rawls, a lo largo de su trabajo filosófico, introduce bastantes modificaciones a su propuesta inicial; pero, su apuesta en todas ellas, no ha sido otra que presentar un sistema justo de cooperación, con unas reglas públicas definidas, que recompensarán a quien acate las condiciones preestablecidas. Por tanto, bajo esta égida, cabe preguntarse: Cómo hacemos entonces para mitigar las desigualdades, ya sean de orden social, natural o de la fortuna. Para Rawls, la respuesta significativa tendría que ser a través de unos principios de justicia como manera equitativa de enfrentar esas arbitrariedades. En su propuesta, Tampoco se puede pasar inadvertida la manera como asocia las ideas tradicionales de libertad, igualdad y fraternidad, con la interpretación democrática de sus dos principios de justicia.
Veamos: "la libertad corresponde al primer principio, la igualdad a la idea de igualdad en el primer principio junto con la justa igualdad de oportunidades, y la fraternidad al principio de diferencia" (Rawls, 2006: 108).

Así las cosas, los principios de justicia no solamente van a mitigar las desigualdades sociales en la estructura social, sino que, además, van a reconciliar el debate filosófico hasta entonces suscitado "libertades versus igualdad", como quiera que estos principios de justicia deberán entenderse como todo un sistema. Así, por un lado, el primero establece la repartición de derechos y de deberes básicos; el segundo, cuenta con las oportunidades abiertas a todos sin distinción alguna y además de una distribución de riqueza que compensa a todos los miembros bajo una cooperación voluntaria de todos sus participantes, en especial, a los menos aventajados, tenemos pues que los dos principios incorporan la idea de libertad e igualdad que deben funcionar en unidad. Ahora bien, la justicia como equidad rawlsiana se enmarca, además, en una concepción igualitarista que pretende regular las desigualdades económicas y sociales, limitar las brechas de clases. Asimismo, considera primordiales los temas como la pobreza, donde unos tienen para abastecerse y otros, sin embargo, no tienen ningún medio para atender sus mínimos. Al respecto, se pregunta Rawls "(...) a menos que haya escasez real, todos deberíamos tener al menos lo suficiente para satisfacer nuestras necesidades básicas" (Rawls, 2017: 177). Es pues, en esta instancia, el tema central y la preocupación que el presente ensayo pretende abordar. En breve, acá se considera que le corresponde al Estado garantizar el cubrimiento de necesidades básicas que posibiliten una vida digna o en otras palabras, entre otras cosas, unas de sus funciones tendrá que ser tomar a su cargo los derechos sociales fundamentales.

A lo largo del escrito, se habrá de presentar una sustentación desde la doctrina contractualista 
para mostrar el papel fundamental que tienen las instituciones para posibilitar el desarrollo de la persona moral. Este último término se entiende aquí bajo el imperativo kantiano de tratar a la humanidad tanto en sí mismo como en los otros, siempre como un fin y nunca simplemente como un medio. Acá se asume que solo a través del Estado se pueden garantizar unos mínimos de bienestar. Esta argumentación hace uso de un enfoque denominado institucionalismo trascendental, cuyo objetivo no es otro que preguntarse por la forma cómo se pueden establecer esquemas institucionales justos. Desde nuestro punto de vista, es a partir de estos esquemas que se permitirá la materialización real de la libertad y los derechos básicos de las personas, entre los que incluimos el cubrimiento de las necesidades básicas, pero, además, me atrevo a afirmar, en caso de su incumplimiento "el Estado habrá roto el pacto", lo que a su vez tendrá efectos a favor de los coasociados, entre los que se destaca el uso de los mecanismos legítimos para hacer cumplir los acuerdos. Aún más, tal incumplimiento nos eximiría de acatar los deberes que el Estado impone cuando se esté en juego el cubrimiento de tales mínimos que posibiliten nuestro desarrollo moral.

\section{Desarrollo}

Cuando una madre se levanta y mira en su cocina que no tiene comida para darle a sus pequeños, que no ha podido acceder a un empleo, que, por sus condiciones naturales, sociales o la fortuna moral, no ha podido gozar de las mismas oportunidades que otros disfrutan, cuando la sociedad en la que se encuentra no tiene políticas que beneficien a las mujeres cabezas de familia y tampoco protecciones especiales para los niños y niñas vulnerables, se habla aquí de una situación de pobreza ${ }^{1}$, donde hay hambre y se carece de un

1 Peter Singer considera que el tema de pobreza no se trata de un problema de escasez, sino de un problema de distribución, trae a colación algunos datos de la pobreza: "Millones de personas pasan hambre continuamente; otras sufren enfermedades carenciales e infecciones que podrían eliminarse con una dieta mejor. Los niños son los más afectados. (...) McNamara ha definido el concepto de pobreza absoluta como unas condiciones de vida que se caracterizan de tal forma por una desnutrición, analfabetismo, enfermedades, entorno miserable, alta mortalidad infantil y una baja esperanza de vida, que se encuen- ingreso para satisfacer las necesidades básicas. Lo que se pretende en este aparte desde la teoría de Rawls, será tratar de dar razones que posibiliten justificaciones en la forma cómo corresponde a las instituciones no solo establecer unos principios de justicia que procuren la mitigación de las desigualdades sociales, sino que, además, establezcan los mecanismos suficientes con los cuales asegurar un mínimo social que cubra las necesidades básicas y así, posibilitar el desarrollo de la persona moral.

Precisamente, en la segunda versión de su teoría de Justicia Distributiva, Rawls analiza algunas consideraciones que apoyan las características del principio de la diferencia, y es, en esta instancia, donde hace su aparición una idea primigenia sobre la manera como los principios de justicia irían a proteger las demandas de la persona moral y aplicar de manera más estricta el principio de la dignidad humana ya planteado por el filósofo alemán, Immanuel Kant. Así, para el filósofo norteamericano: "Porque en esa situación los individuos tienen igual representación en cuanto personas morales que se miran a sí mismas como fines, y los principios que ellas aceptan estarán diseñados racionalmente para proteger las demandas de su persona" (Rawls, 1973: 72).

De tal suerte, el principio de diferencia reza de la siguiente manera: cuando se renuncia a ganancias que no redunden en las expectativas de los menos aventajados, se dará lugar a tratar a las persona como fines en sí mismos, dado que tratarlas como medio, sostiene Rawls, "significa estar dispuesto a imponerle perspectivas más bajas de vida en aras de compensar suficientemente las expectativas mayores de otros y obtener así una suma más elevada de ventajas" (Rawls, 1973: 72). Será, por lo tanto, nuestro valer ${ }^{2}$ considerado como el bien primario más

tra por debajo de cualquier definición razonable de decencia humana" (Singer, 2003: 171-172).

2 En las últimas versiones de la Teoría de Rawls, cuando elabora una lista normativa de los bienes sociales primarios, se incorporan las bases sociales del autorrespeto: "con lo que entendemos aquellos aspectos de las instituciones básicas normalmente esenciales si los ciudadanos han de tener clara conciencia de su valor como personas y han de ser capaces de promover sus fines con autoconfianza" (Rawls, 2017: 92) 
importante de la sociedad, constituido, en gran parte, por el respeto mutuo que los ciudadanos se tienen como personas morales.

Sin embargo, el propio valer no solo depende del respeto que surja entre los coasociados, también estará determinado por el avance de nuestra civilización. Así, A decir de Rawls, “El perfeccionamiento de las instituciones políticas ${ }^{3}$ elimina la oposición de intereses y las barreras y desigualdades que alientan a los ciudadanos a hacer caso omiso de las demandas reciprocas" (Rawls, 1973: 76). Sostiene pues que, solo a través de las instituciones, es posible que se considere no solo el beneficio individual, sino, también, el general. Desde el punto de vista rawlsiano, con lo anterior se le podría dar respuesta a uno de los interrogantes fundamentales de la teoría contractualista, a saber: ¿es posible cooperar?

Con esta pregunta entonces, se tendrá una justificación con la cual avalar la tesis que sostiene que todo desarrollo moral se podría propiciar a través del perfeccionamiento de las instituciones políticas. Esta tesis puede sostenerse desde la teoría contractualista a la manera de una contraprestación que surge entre las partes, tanto del Estado como de sus coasociados, para dar cumplimiento a los pactos establecidos. Justamente, en Teoría de la Justicia, Rawls ha buscado primero definir las concepciones de justicia de sus instituciones; para luego definir los principios de las personas y por último, lo que respeta al derecho internacional.

En cuanto a la preocupación que acá nos atañe, los principios de las personas deben ser considerados desde el principio de imparcialidad. Este último se puede definir como el cumplimiento por parte de los individuos de las diferentes reglas y deberes dentro de la institución. Es así que cuando se cumple con ciertas exigencias, primero se podría afirmar que se trata de una sociedad justa, esto es, que cumple con los dos

3 De aquí surge la idea intuitiva del nombre del capítulo "perfeccionamiento de las instituciones políticas como posibilidad del desarrollo de la persona moral". principios de justicia y a su vez, se aceptan los beneficios del acuerdo o se alcanzan beneficios a partir de tales oportunidades.

De lo anterior se desprende que se trata de una doble contraprestación; es decir, no se pueden recibir ventajas dentro de la empresa cooperativa, sino se cumple con los propios deberes. Bajo estos requisitos, mi obligación dentro del contrato se presenta de manera condicionada, esto es, este deber también se constituye para el Estado mismo. En ese sentido, por ejemplo, si se trata de instituciones injustas, la aceptación de actos voluntarios, no llegan a ser requisitos que se logren alcanzar bajo un régimen arbitrario, como quiera que la estipulación de una obligación contractual, no llega a ser vinculante bajo la tutela de regímenes injustos.

Por mi parte, acá se sostiene que, en todo acuerdo inicial, el Estado debe garantizar unos mínimos de bienestar, y que elevados a rango constitucional, le serán exigibles; así, dado su incumplimiento "el Estado habrá roto el pacto". Justamente, cuando en esa doble contraprestación, no haya unas condiciones mínimas de justicia razonables que cubran las necesidades básicas, traerá como consecuencia que se pueda estipular el incumplimiento de las obligaciones del Estado y por consiguiente, se le podrán otorgar a los coasociados los mecanismos jurídicos necesarios para la exigencia de tales derechos o, en su defecto, cuando no se cuente con tales mecanismos, este estado de cosas nos llegará a eximir del acatamiento de los deberes propios que el Estado ha impuesto. Es decir, este estado de cosas excepcional no podría ser otro que la incapacidad institucional evidente del cubrimiento propio de tales mínimos que posibilitan nuestro desarrollo moral.

Se podría una contextualización más práctica de todo lo anterior, sobre todo cuando se trata de países pobres en los queno se ha dado un desarrollo de los derechos sociales constitucionales, ni 4.Rawls, tratare este aspecto como una doble tensión del compromiso. 
mucho menos una mínima satisfacción de las necesidades básicas. Bajo tales condiciones, se encuentran altas tasas de pobreza, desempleo y seguridad social no cubierta. Desde nuestro punto de vista, es en esta instancia donde las estructuras políticas y sociales requieren ampliar el margen de derechos constitucionales o en su defecto, materializarlos. O en otras palabras, el Estado debe dar cumplimiento a la parte del acuerdo que le compete, esto es, este debe hacer todo lo posible para garantizar unos principios de justicia que permitan unos mínimos razonables para los ciudadanos y posibiliten el desarrollo del sujeto moral.

Mantengámonos en este contexto de los países poco desarrollados del ejemplo inicial. En esta instancia, cabría preguntase si la única opción que tendría una madre para conseguir el alimento necesario para su progenie no sería sino recurrir al hurto, a la prostitución o acudir a cualquier otro tipo de acción ilícita. Desde un contexto moral, y en relación con el objeto de estudios que nos atañe, se asumirá que esta madre ha adoptado una concepción de bien deontológica dentro de su esfera individual de acción, así su máxima tendría que ser "no robar" ni "instrumentalizar su cuerpo" en ninguna circunstancia. No obstante, se podría inferir que ante la gravedad de la situación antes descrita, a esta madre poco o nada le tendría que importar seguir amparándose bajo su concepción moral, toda vez que la prioridad de obtener alimento para sus hijos será más apremiante. En ese sentido, cuando el Estado no garantiza o ha sido incapaz de garantizar unos mínimos de bienestar, la posibilidad del desarrollo de la persona moral y la libertad de esta madre le ha sido negada, pues, bajo estas condiciones no tiene ninguna opción.

Este caso visto desde una perspectiva institucional, permitiría afirmar que el Estado no ha dado cumplimiento al pacto, como quiera que no ha logrado garantizar unos derechos sociales mínimos que le confieran un vivir digno a los individuos que hacen parte de tal comunidad política. Asimismo, las consecuencias desafortunadas también las tendría que padecer el Estado, ya que, fuera de las exigencias estatales legítimas que tiene que acoger para sí cada individuo, se podría agregar que, sin mecanismo de acción, los individuos no tendrían una obligación de acatar cargas o deberes impuestos por un régimen injusto. Esto es, el Estado perdería su legitimidad para sancionar acciones ilícitas llevadas a cabo por aquellas personas en situación de carencia, que no tengan posibilidad de elección y que procuren garantizarse unos mínimos de bienestar social.

A partir de lo anterior, nuestra hipótesis propone la necesidad de concebir una teoría política fundada en unos principios de justicia que busquen garantizar un mínimo social dentro de la estructura social. Es así que, una vez estos principios hayan sido establecidos al nivel de rango constitucional, adquirirán un carácter vinculante suficiente y posibilitarán el desarrollo de la persona moral. Asimismo, cualquier incumplimiento tendría que hacer legítima cualquier demanda de los coasociados perjudicados.

En defensa de esta idea, a saber, de que solo a través de una concepción institucionalista se posibilitaría el desarrollo de la persona moral en sociedad; acá se considera como buena fuente de fundamentación algunas tesis contractualistas, y uno de sus pilares del estado: su poder coercitivo. Justamente, sin el surgimiento del Estado, no se podría garantizar el cumplimiento de derechos naturales, ya que, solo a través de su capacidad de coerción se podría garantizar su materialización y de manera razonable, igualmente, la vinculación de las partes a los acuerdos.

Como bien se sabe, la tesis principal del contractualismo no es otra que la transición de un estado de naturaleza a uno civil. En este 
primer momento, a partir del ejercicio racional, todo individuo podría aceptar la existencia de unas leyes de la naturaleza que, sin embargo, no ofrecerían ninguna posibilidad de prosperidad material, social o moral. Es, en esta instancia, donde todo sujeto racional encuentra la necesidad de alcanzar justicia y protección. Es allí donde tendría que surgir la necesidad de un pacto que de origen a unos derechos mínimos y a un poder vinculante que lleve a todos los involucrados a cumplirlos. En toda teoría contractualista, este poder vinculante solo se presenta con la figura del Estado.

Así las cosas, las leyes de la naturaleza se tendrían que considerar en el ámbito de lo razonable, pues proponen una serie de virtudes que inclinan a los individuos a la cooperación y al orden social; no obstante, solo obligan en conciencia. Precisamente, por razones prudenciales, para todo individuo en estado de naturaleza, sería irracional someterse a sus preceptos cuando no hay garantía alguna de que todos los demás también lo habrían de hacer. Es pues, allí, donde se requiere de la creación de un Estado con el poder suficiente de coacción. Desde el punto de vista contractualista, solo de este modo, sería racional acatar cualquier precepto que pretenda garantizar derechos y deberes que posibiliten alcanzar una convivencia en sociedad.

Ahora bien, habría que insistir en que muchas, si no todas las teorías contractualistas concuerdan en la afirmación de que en el estado de naturaleza no se lograría ningún nivel de justicia, ni cumplimiento a las leyes de la naturaleza, a no ser, bajo la premisa del beneficio mutuo. Sería así y solo así, bajo el principio del beneficio común, que se harían posibles acuerdos y la existencia de un poder común que obligue a los pactantes a cumplir lo pactado.

Así, el fundamento de todo sistema social tendría que residir, por un lado, en que se renuncie voluntariamente a la libertad natural, cuyo límite no sería otro que la fuerza individual o la suma de individuos; pero, por el otro, tal renuncia, le conferiría a los pactantes una libertad civil y el aprovechamiento efectivo de derechos. Igualmente, tendría que ser posible la consecución de una libertad y una autonomía moral y política. En breve, desde el punto de vista contractual, el contrato social tendría que posibilitar el surgimiento del Estado, del poder político y el establecimiento de unos derechos y deberes para cada una de las partes y sobre todo, un reconocimiento efectivo de la persona moral.

La defensa de nuestra tesis se fundamenta así en la teoría contractualista, sin embargo, es importante revisar algunas críticas que se le han planteado a dicha teoría. En primer lugar, se quiere destacar la presentada por Michael J. Sandel en su texto Justicia ¿Hacemos lo que debemos? Allí, plantea que, puesto que ningún estadounidense ha firmado un contrato social, cabría preguntarse entonces: ¿cómo podemos estar obligados a acatar la Ley? Al respecto, afirma que esta obligación ha partido de un consentimiento tácito, a la manera de John Locke. Sin embargo, este contrato no podría alcanzar la categoría de auténtico. Por su parte, Sandel sostiene que Immanuel Kant ha recurrido al consentimiento hipotético para dar respuesta a la pregunta planteada con anterioridad y afirma: "Una ley es justa si la sociedad en su conjunto, de haber podido, la hubiese refrendado (...) ¿Cómo podría un acuerdo hipotético ejecutar la tarea moral de uno real?" (Sandel, 2013: 161).

Si bien en Rawls se propone un acuerdo hipotético en una situación originaria de igualdad, en aras de escoger unos principios de justicia; para Sandel, el interrogante sigue sin respuesta ya que, a la respuesta rawlsiana cabría plantearle otro interrogante, a saber: ¿cómo es posible que los principios de justicia se deriven de un acuerdo que nunca sucedió en realidad? Asimismo, también ha de quedar la siguiente duda: ¿cómo podemos lograr que el acuerdo aceptado sea 
justo? Según Sandel, "Que el pueblo ratifique una constitución no prueba que lo que promulga sea justo. Piénsese en la Constitución de Estados Unidos de 1787. Pese a sus muchas virtudes, tenía el defecto de aceptar la esclavitud, y así fue hasta después de la guerra civil" (Sandel, 2013: 164). Así las cosas, la aceptación del acuerdo no hace que este pueda ser o sea justo. Para dar mayor justificación a esta aseveración, Sandel propone un análisis del papel que tiene el consentimiento para crear una obligación, pero, además, el peso moral que tienen los contratos bajo dos ideales: "la autonomía y la reciprocidad". Para este:

En tanto actos voluntarios, los contratos expresan nuestra autonomía; las obligaciones que crean tienen peso porque nos las imponemos a nosotros mismos, porque cargamos con ellas libremente. En tanto instrumentos para el beneficio mutuo, los contratos beben del ideal de la reciprocidad; la obligación de cumplirlos procede de la obligación de pagar a otros por los beneficios que nos aportan (Sandel, 2013: 166).

Empero, la realidad jurídica frente a la doctrina de los contratos hace evidente sus propias dificultades y que no pueden resolverse con facilidad. A decir de Sandel, la idea general es simple: a través del consentimiento surge la obligación, pero, entonces, no importa si se le saca provecho o no, a la contraprestación. Desde esta óptica, se podría inferir que muchos acuerdos no reportan beneficios; o, por el contrario, puede que no haya consentimiento y se genere una obligación. Sandel lo presenta en términos prácticos con el siguiente ejemplo: considérese el caso de un fontanero que le cobró a una anciana unos 50.000 mil dólares por arreglar un retrete, lo cual evidentemente desborda los límites del mercado y de la justicia. Para Sandel, del hecho de que haya habido un acuerdo previo, no implica que lo pactado haya llegado a ser equitativo. Además, agrega, el mero consentimiento no basta para crear obligaciones morales.
Según el filósofo norteamericano, los acuerdos reales pueden carecer de estos ideales de “autonomía y reciprocidad". Es así que resulta difícil imaginarse un contrato perfecto, es decir, en el que no haya ventajas, coacción o que una de las partes no se encuentre en una mejor posición de negociación. De lo anterior, se podría inferir que, dado este estado de cosas, las diferencias de las partes tanto de su conocimiento como de su poder negociador, haría muy difícil el acceso a posiciones de igualdad. Según Sandel: "Por eso, los contratos reales no son instrumentos morales autosuficientes" (Sandel, 2013: 172).

La crítica de Sandel a la teoría del contractualismo se presenta en dos frentes. Veamos. En primer lugar, afirma que el contrato social no sucedió en realidad, y en segundo lugar, el consentimiento no llega a constituirse en condición suficiente para la obligación moral. Plantea que podría pensarse en un beneficio real sin que haya mediado con antelación consentimiento alguno.

Desde nuestro punto de vista, se considera que el problema que se da en torno al contrato social no radica en el interrogante que se inquiere sobre el carácter real o hipotético del contrato. De hecho, se considera que la preocupación real no sería otra que interpelarse por el objetivo del contrato. Precisamente, para el contractualismo clásico, el contrato no tiene otra pretensión que dar origen al Estado. No obstante, cabe ser muy cautos con lo anteriormente planteado, toda vez que, para nuestros estados modernos, ese objetivo no deja de ser insuficiente. Uno de nuestros grandes problemas políticos es la legitimad. Justamente, la idea del contrato ha llegado a ser uno de los más fuertes argumentos en la filosofía política para conferir legitimidad a un sistema social y jurídico. Además, se ha constituido en una fuente muy efectiva para deslegitimar aquellas estructuras sociales que no concuerdan con una fundamentación razonable y justa. En el caso particular de esta investigación, se ha pretendido evidenciar la necesidad de incorporar 
unos mínimos que permitan una vida digna como fundamento de una sociedad justa. Una vez alcanzados estos mínimos, el Estado debe garantizarlos con el fin de alcanzar y mantener su legitimidad.

En relación con la segunda crítica planteada por Sandel, cabe llamar la atención sobre el hecho de que el filósofo norteamericano fundamente la obligación en la idea de un beneficio recibido sin que haya mediado consentimiento alguno. Al respecto se podría ejemplificar esta idea con los trabajadores informales que buscan ganarse el sustento limpiando parabrisas en las calles. De nuestra parte, se podría contemplar la posibilidad de una sociedad fundada en la idea de una obligación que se crea a partir del beneficio recibido. Es decir, el principio de cooperación no tendría que estar mediado por un acuerdo o un consentimiento vinculantes, más bien, por una idea de servicio a los demás.

A partir de lo anterior se ha pretendido contextualizar la doctrina del contrato social y su objetivo general. Ahora bien, para Rawls, el papel del contrato no va a ser otro que justificar la idea de una sociedad como un sistema equitativo de cooperación. De este, no se pretende que surja una forma de gobierno, sino el establecimiento de unos principios de justicia que estipulen una estructura básica. Este primer acuerdo se celebra entre personas racionales, libres e iguales, bajo la idea de un velo de ignorancia que permitiría que cada persona que pacte alcance cierto nivel de neutralidad, es decir, eliminar posiciones ventajosas bien sea en las condiciones naturales, sociales, o fortuna. Este velo, según Rawls, crearía las condiciones necesarias para alcanzar un acuerdo equitativo y además, generaría un sentido de justicia lo suficientemente vinculante y legítimo para que todos encuentren razones suficientes para acatar los principios alcanzados. En síntesis, se debe contar no solo con una teoría razonable, sino, además, con un poder estatal lo suficientemente vinculante. Para Rawls: "el poder político es siempre, obvio decirlo, poder coercitivo respaldado por la maquinaria del Estado para hacer cumplir sus leyes" (Rawls, 2017: 243). Cabe agregar que, en un régimen constitucional, el poder político también está representado por los ciudadanos libres e iguales.

Ahora bien, en Rawls, resulta importante contextualizar la idea de las personas libres e iguales. Esto se debe hacer dada la necesidad de identificar tanto las obligaciones como las exigencias de cada una de las partes al vincularse a un contrato. Desde el punto de vista del filósofo norteamericano, las personas que participan en un acuerdo cooperativo poseen dos facultades morales. Por un lado, cuentan con un sentido de la justicia y por el otro, una concepción del bien. $\mathrm{Al}$ respecto, afirma que:

\begin{abstract}
Al decir que concebimos a las personas como poseedoras de las dos facultades morales, estamos diciendo que tienen las capacidades requeridas no solo para participar en una cooperación social mutuamente beneficiosa durante toda una vida, sino, también, sentirse motivados a honrar los términos equitativos de dicha cooperación como fines en sí mismos (Rawls, 2017: 44).
\end{abstract}

Estas dos facultades, dirá Rawls, definirán lo que es la persona moral. Este concepto pertenece a una concepción normativa y política que se desprende de su pertenencia a una sociedad democrática y se encuentra definida en sus textos fundamentales. Así pues, estas facultades morales se constituirán en la base de la igualdad política, cuyo objetivo no será otro que la participación activa en una sociedad que vincula coercitivamente a las instituciones con no otro fin que lograr la aplicación de la justicia de acuerdo con las reglas definidas. Así, para Rawls, la distinción entre la sociedad democrática y la comunidad tendría que ser la siguiente: “(...) solo la sociedad, con su forma política de gobierno y su ley, ejerce poder coercitivo" (Rawls, 2017: 45). Hasta aquí, la presente investigación se 
identifica con la necesidad de una potestad de un Estado coercitivo que garantizaría no solo la vinculación sino el cumplimiento del acuerdo cooperativo.

De otra parte, distingue Rawls, el sentido libre de los ciudadanos, en un primer aspecto, lo identifica con "la facultad moral de poseer una concepción del bien" (Rawls, 2017: 46), la cual estipula que cada ciudadano pueda cambiar, reformular o adoptar la postura que más se acomode a sus ideales. Sin embargo, estos valores de justicia adoptados por los ciudadanos se deben ajustar, reconciliar $y$ materializar a través de las instituciones. Al respecto, afirma Rawls: "Y en una sociedad bien ordenada apoyada en un consenso entrecruzado, los valores y compromisos políticos (más generales) de los ciudadanos, que forman parte de su identidad no institucional o moral, son prácticamente los mismos" (Rawls, 2017: 48). Agregar que tampoco se podría olvidar que las exigencias de los ciudadanos al Estado, solo resultan válidas en tanto se encuentren incluidas en una concepción política de justicia. En ese sentido, Rawls llamará a tales exigencias:

(...) fuentes autentificatorias de exigencias válidas. (...) pues siempre que las concepciones del bien defendidas por los ciudadanos sean compatibles con la concepción publica de la justicia, esos deberes y obligaciones serán autoautentificatorios desde un punto de vista político (Rawls, 2017: 48).

Así las cosas y ante la concepción de una connotación doble del acuerdo, se tendría, por un lado, un poder coercitivo que encarna el Estado con el cual se pretende garantizar los deberes y las obligaciones de las partes, a saber: los principios de justicia que garantizan unos mínimos de bienestar. Y, de otro lado, se presentan unas exigencias válidas de los ciudadanos que se han de materializar a través de acciones tendientes a exigir del Estado el cumplimiento de los acuerdos cooperativos pactados y elevados a rango constitucional. Desde nuestra investigación, se sostiene que tales garantías, solo resultarían posible desde la figura del institucionalismo que bajo la idea del perfeccionamiento de las instituciones políticas logre establecer unos mínimos que posibiliten el desarrollo de la persona moral. Esta doctrina se conoce como institucionalismo trascendental.

Robert Alexy hace un análisis de la teoría discursiva del derecho; así, en relación con la institucionalización, hace evidente la necesidad de una teoría del Estado y del Derecho bajo un fundamento filosófico. Según Alexy, esta necesidad surge de la debilidad de un discurso práctico que no logra obtener la plena aprobación. De hecho, acota que aquellas normas que se contradicen, no podrían llegar a ser vinculantes y más cuando se trata de conflictos sociales. Al respecto, sostiene que:

Este argumento hobbesiano se complementa con uno kantiano. Este afirma que en un discurso racional no se puede justificar cualquier sistema jurídico, sino solo aquellos que llenen las elementales exigencias de la razón práctica. Entre estas se cuentan las garantías de los derechos humanos fundamentales y la institucionalización de procedimientos democráticos y conformes con el Estado de Derecho (Alexy, 1995: 54).

En este orden de ideas, se ha pretendido seguir con la misma línea argumentativa. Así, por un lado, se tiene un acuerdo que reviste fuerza y coacción; pero, además, una legitimidad que se funda en la garantía que logre conferirles a los mínimos sociales dentro de una concepción política de justicia. Por otro lado, acá se considera que una gran ventaja del institucionalismo es que, solo en la medida que se tengan instituciones políticas preocupadas por garantizar unos principios de justicia que brinden unos mínimos de bienestar que posibiliten una vida digna, sería posible materializar los derechos. Rawls acepta este 
postulado solamente en las últimas versiones de su teoría. Sostiene que:

Este principio puede ir precedido por un principio léxicamente anterior que exija que queden satisfechas las necesidades básicas, al menos, en la medida en que su satisfacción es una condición necesaria para que los ciudadanos entiendan y sean capaces de ejercer fructíferamente los derechos y libertades básicos. (...) (Rawls, 2017: 75).

Para finalizar este aparte, se advierte que Rawls hace una muy breve alusión al problema acá señalado, a saber: en la necesidad de establecer unos principios de justicia que garanticen unos mínimos de bienestar, que posibilitarán a los ciudadanos el ejercicio de sus derechos y el desarrollo de su persona moral. Es así que, una vez que cada individuo encuentre satisfechos estos mínimos, se posibilitará que cada cual pueda asumir sus otros compromisos de acuerdo con sus fines generales, pero, además, se hagan responsables de sus acciones. También las instituciones políticas obtendrán cierta credibilidad en tanto se mantengan fieles a los compromisos defendidos por los ciudadanos. Es decir, a partir de lo anterior, el Estado constitucional democrático y su concepción pública de justicia podrán alcanzar plena legitimidad.

\section{Conclusiones}

Mi preocupación ha girado en torno a la concepción igualitarista de Rawls cuyo objetivo no es otro que regular el problema de las desigualdades económicas y sociales, en especial, lo relacionado con la idea de unos mínimos que permitirían llevar una vida digna. En esta investigación se ha partido de la idea de un diseño institucional con el cual se pudiera garantizar la constitucionalización de unos mínimos sociales, en vez de un principio de diferencia como lo plantea la propuesta de Rawls. Justamente, problemas tan acuciantes como la pobreza, han hecho que se tengan que concebir, proponer y considerar con urgencia medidas que no solo busquen mitigar, sino cubrir necesidades básicas tanto de individuos como de comunidades enteras. Asimismo, esta propuesta ha considerado la posibilidad de mecanismos que posibiliten acciones legitimas para que los ciudadanos puedan exigirle al Estado el cumplimiento de su compromiso fundamental de asegúrale a cada cual un mínimo; así, una vez éstas resulten efectivas, cada ciudadano podrá hacer uso de los demás ejercicios de la vida política.

A partir de nuestra tesis principal, se ha pretendido justificar la idea de una teoría contractualista de nuevo cuño. Varios han sido nuestros argumentos. Veamos. Desde nuestra óptica, un acuerdo inicial entre las partes deberá decidir sobre el establecimiento de una obligación a partir de la cual el Estado tenga que proveer a sus coasociados unos mínimos sociales. Esta exigencia deberá tener rango constitucional y sus consabidos mecanismos legales de protección y exigibilidad. La razón principal de esta exigencia radica en la necesidad de que una sociedad bien ordenada asuma, como una de sus funciones fundamentales, posibilitar el desarrollo de la persona moral y política.

A reglón seguido, y una vez estipulado lo anterior, cualquier ciudadano podrá aducir legalmente que el Estado ha incurrido en un incumplimiento del pacto, y, por lo tanto, este podrá acudir a los mecanismos jurídicos estipulados para hacer que el Estado asuma su función. Dado el caso de que no se contase con ningún tipo de mecanismos jurídicos de protección o exigibilidad, el ciudadano se podría considerar eximido de sus deberes propios para con el Estado. En suma, nuestra propuesta ha buscado argumentar sobre la necesidad de concebir una teoría política que se fundamente en el perfeccionamiento de unas instituciones y el establecimiento de unos principios de justicia que busquen garantizar a cada ciudadano un mínimo social dentro de la estructura social. 
En esta instancia se quisiera resaltar el análisis que se ha hecho sobre el incumplimiento del Estado para con sus coasociados y las implicaciones jurídicas que se pudieran desencadenar ante tal incumplimiento. Cabe resaltar esta idea como uno de los grandes aportes de la teoría contractualista a la discusión política actual. Asimismo, se ha querido hacer evidente que el incumplimiento por parte del Estado de sus obligaciones podría desembocar en una disminución o pérdida de su legitimidad y por tanto, de sus fundamentos legales como instancia sancionadora de hechos ilícitos perpetrados por personas en situación de carencia y sin ninguna otra opción para procurarse unos mínimos de bienestar social.

Otro de los argumentos que apoyan esta propuesta se fundamenta en uno de los pilares del Estado: su poder coercitivo. La tesis principal del contractualismo no es otra que la transición de un estado de naturaleza a uno civil. Así, una vez en situación de civilidad, las partes pactantes se vinculan mutuamente a los compromisos acordados a través de la figura del Estado y su poder de hacer que los pactos sean vinculantes.

Ahora bien, esta propuesta fundamentada en la teoría contractualista y cuya crítica principal es la de un contrato social que nunca sucedió, hace que nuestra investigación considere dos aspectos. Veamos. Por una parte, hemos de dejar de lado el hecho de que el contrato haya sido real o hipotético. Por otra parte, nuestra real preocupación sería determinar el objetivo del contrato. Precisamente, es un hecho que las doctrinas clásicas del contrato han tenido como papel esencial explicar el surgimiento del poder y del Estado. Por su parte, en los Estados modernos, el problema ha sido fundamentalmente el de la legitimidad y su margen de maniobrabilidad. En consecuencia, la gran preocupación de la filosofía política contemporánea ha sido la legitimidad y aquellos aspectos que le confieren existencia y estabilidad a un sistema social y jurídico. En tal caso, aquellas estructuras sociales que no se encuentren en el marco descrito y prescrito por una fundamentación razonable y justa habrán de ser consideradas ilegítimas e inviables. En nuestro caso particular el objetivo ha sido ofrecer argumentos para evidenciar la necesidad de incorporar unos mínimos que le permitan a los coasociados alcanzar una vida digna. Asimismo, se han dado las razones suficientes para que parte de la legitimidad del Estado radique en los mecanismos que este despliegue para dar cumplimiento y protección a este derecho.

Bajo esta égida, a lo largo de la presente investigación se ha desarrollado un análisis crítico del contrato social en Rawls. Se ha visto que esta propuesta pretende alcanzar un sistema equitativo de cooperación cuyo fundamento es el de proponer unos principios de justicia para constituir una estructura social. Según Rawls, el contrato se celebra entre personas racionales, libres e iguales, bajo condiciones de equidad, para generar una vinculación legítima. Así, una vez alcanzado un pacto, se identifica la necesidad de un Estado coercitivo que garantizaría no solo la vinculación sino el cumplimiento del acuerdo cooperativo. Además, a través de este análisis de la propuesta rawlsiana, se han pretendido hacer evidentes las obligaciones de las partes que se han vinculado al contrato a partir de su facultad moral. Esta última consta de dos partes, por un lado, un sentido de la justicia y por el otro, una concepción del bien. Es así que las exigencias solo serán válidas en la medida que las concepciones de bien se identifiquen con una concepción pública de justicia.

Aquí se considera que el institucionalismo presenta una gran ventaja, a saber: solo en que se tengan instituciones políticas preocupadas por garantizar unos principios de justicia que brinden unos mínimos de bienestar que posibiliten una vida digna, sería posible materializar los derechos. Rawls acepta este postulado solamente en las últimas versiones de su teoría. 
En definitiva, a lo largo de estas líneas, se ha buscado argumentar críticamente que el Estado debería garantizar unos mínimos sociales a nivel institucional y que, una vez satisfechos, por un lado, coadyuva en el alcance de una vida digna para cada cual y por ende, asegura que cada individuo pueda asumir otra serie de compromisos de acuerdo con sus propios fines $\mathrm{y}$ por otro, crea las condiciones necesarias para que cada persona se haga responsable en la toma de decisiones políticas y sus posibles consecuencias. Por su parte, a nivel institucional y una vez sean honrado los pactos, todo este entramado institucional permitirá legitimar la concepción política de justicia de la estructura social o en su defecto, se tendrán argumentos de peso suficiente para que se tomen las respectivas medidas y sean incorporadas dentro del rango constitucional.

\section{Referencias bibliográficas}

Alexy, R. (1995). Teoria del discurso y derechos humanos. Colombia: Universidad Externado de Colombia.

Amor, C. (2006). Rawls post Rawls. Buenos Aires: Universidad Nacional de Quilmes.

Arango, R. (2012). El concepto de derechos sociales fundamentales. Colombia: Legis.

Berlin, I. (2008). Sobre la libertad. España: Alianza editorial.

Dieterlen, P. (2013). La pobreza: Un estudio filosófico. Mexico: Fondo de cultura economica.

García, J. I. (1985). La Teoria de la Justicia de John Rawls. Madrid: Centro de Estudios Constitucionales.
Hayek, F. (1998). Los fundamentos de la libertad. Madrid: Unión editorial.

Hobbes, T. (2011). Leviatán. España: Fondo de Cultura Economica.

I.M.Crombie. (1990). Analisis de las doctrinas de Platón. Madrid: Alianza Universidad.

Locke, J. (1995). Segundo tratado sobre el gobierno civil. Barcelona: Altaya S.A.

Mill, J. (1991). Sobre la libertad y comentarios a Tocqueville. Madrid: Espasa Calpe.

Mora, F. (202). Diccionario de Filosofia. España: Ariel. S.A.

Moreso, J. M. (2006). Rawls post Rawls. Buenos Aires: Universidad Nacional de Quilmes.

Nussbaum, M. C. (2002). Las mujeres y el desarrollo de lo humano. España: Herder.

Platon. (1984). CRITÓN. Medellín: Bedout S.A.

Rawls, J. (1973). justicia distributiva. Economic Justice, 319-362.

Rawls, J. (1995). Liberalismo Politico. Mexico: FCE, UNAM.

Rawls, J. (1999). Justicia como equidad. Espana: Tecnos.

Rawls, J. (2006). Teoría de la Justicia. México D.F: Fondo de Cultura Económica.

Rawls, J. (2006). Teoría de la Justicia. México: Fondo de Cultura Económica.

Rawls, J. (2007). Lecciones sobre la historia de la filosofia moral. España: Paidos. 
Rawls, J. (2009). Lecciones sobre la historia de la filosofia política. Madrid, España: Paidos Iberica, S.A.

Rawls, J. (2017). La justicia como equidad una reformulación. España: Paidos.

Rousseau, J. (2012). El Contrato Social. México: Taurus.

Sandel J., M. (2013). Justicia Hacemos lo que debemos? Colombia: Debate.

Sen, A. (2014). La idea de la Justicia. Bogotá, Colombia: Taurus.

Sen, M. C. (1993). La Calidad de Vida. Mexico: Fondo de Cultura Economica.

Singer, P. (2003). Etica práctica. España: Cambridge University Press.

Thoreau, H. D. (2011). Sobre la desobediencia civil. Medellin, Colombia: Universidad de Antioquia.

Wolff, R. P. (1977). para comprender a Rawls una reconstrucción y una cirtica de la teoria de la justicia. Mexico: Fondo de Cultura Economica. 